\title{
ROLE OF BREAST ULTRASOUND IN EVALUATION OF BIRADS 3 AND BIRADS 4 BREAST MASSES
}

\author{
Adarsh A. D1, Krishna Kumar R2, Venugopal Kodumur ${ }^{3}$, Manas Kumar Bora ${ }^{4}$ \\ ${ }^{1}$ Assistant Professor, Department of Radiology, Aarupadai Veedu Medical College and Hospital, Pondicherry. \\ ${ }^{2}$ Associate Professor, Department of Radiology, Aarupadai Veedu Medical College and Hospital, Pondicherry. \\ ${ }^{3}$ Senior Resident, Department of Radiology, Aarupadai Veedu Medical College and Hospital, Pondicherry. \\ ${ }^{4}$ Professor, Department of Radiology, Aarupadai Veedu Medical College and Hospital, Pondicherry.
}

\begin{tabular}{l}
\hline ABSTRACT \\
\hline BACKGROUND \\
Breast Ultrasound (US) is widely used as a diagnostic tool in evaluating mammographically detected masses, ${ }^{(1)}$ palpable lumps, ${ }^{(2,3)}$ \\
nipple discharge ${ }^{(4)}$ and in guiding percutaneous biopsy.(5) Breast ultrasound is a primary diagnostic tool along with mammography \\
for the evaluation of breast masses. \\
Aim- To determine the accuracy of Breast Ultrasound in evaluating BI-RADS 3 and 4 breast masses with pathologic diagnosis as \\
reference gold standard.
\end{tabular}

\section{MATERIALS AND METHODS}

Study was conducted in the period, December 2015 to December 2016. A total of 756 patients who presented to the Surgery outpatient department with complaints of breast lump, pain or nipple discharge were subjected to breast imaging. From 756 patients, 143 patients who had BIRADS 3 and 4 mass were selected for the study. Patients with masses that fulfilled the BI-RADS 3 and 4 were enrolled in the study. Breast ultrasound with and without Doppler performed in these patients with GE Voluson S6 (Linear Array 4 - $12 \mathrm{MHz}$, Curved Array 2 - $8 \mathrm{MHz}$ ). Informed written consent for the ultrasound-guided biopsy procedures was obtained from each patient.

\section{RESULTS}

All the 143 breast masses were evaluated and categorised according to the BI-RADS classification on B mode US. A total of 51 masses were categorised into BI-RADS 3 and 92 masses into BI-RADS 4. Among the 51 BI-RADS category 3 masses, only 4 (7.8\%) were malignant. Of the 92 masses of category 4, 75 (81.5\%) were malignant on histopathology. Thus, the B mode US had a sensitivity of $94.9 \%$ and specificity of $73.4 \%$, positive predictive value of $81.5 \%$ and negative predictive value of $92.2 \%$; (P value $<0.001$ ) in detecting malignancy.

\section{CONCLUSION}

Breast ultrasonography is an appropriate tool in the detection of cancer and should be included in the workup of symptomatic breast disease. Breast ultrasound has a very high sensitivity in detecting malignancies and guiding further management of the suspicious lesions. But the occurrence of false positive diagnosis in the clinical settings like that of inflammatory breast disease should also be considered and the management be decided after correlation with the clinical data and the biopsy results.

\section{KEYWORDS}

Contents of Appendix, Ultrasonography, Predominant Contents.

HOW TO CITE THIS ARTICLE: Adarsh AD, Kumar KR, Kodumur V, et al. Role of breast ultrasound in evaluation of BIRADS 3 and BIRADS 4 breast masses. J. Evolution Med. Dent. Sci. 2017;6(45):3524-3527, DOI: 10.14260/Jemds/2017/760

\section{BACKGROUND \\ Breast Ultrasound (US) is widely used as a diagnostic tool in evaluating mammographically detected masses,(1) palpable lumps, $(2,3)$ nipple discharge ${ }^{(4)}$ and in guiding percutaneous biopsy.(5) Breast ultrasound is a primary diagnostic tool along with mammography for the evaluation of breast masses.}

\section{Aims and Objectives}

AIM- To determine the accuracy of Breast Ultrasound in evaluating BI-RADS 3 and 4 breast masses with pathologic diagnosis as reference gold standard.

Financial or Other, Competing Interest: None.

Submission 28-04-2017, Peer Review 23-05-2017,

Acceptance 30-05-2017, Published 05-06-2017.

Corresponding Author:

Dr. Krishna Kumar $R$

\#11, Priyadarshini Street,

Moogambighai Nagar, Reddiyarpalayam,

Pondicherry-605010, India.

E-mail: drkrishnar1980@gmail.com

DOI: $10.14260 /$ jemds/2017/760

\section{(c) (i) $\$$}

\section{MATERIALS AND METHODS}

Study Type

\section{Observational Diagnostic Study}

Study was conducted in the period, December 2015 to December 2016. A total of 756 patients who presented to the Surgery Outpatient Department with complaints of breast lump, pain or nipple discharge were subjected to breast imaging. From 756 patients, 143 patients who had BIRADS 3 and 4 mass were selected for the study. Patients with masses that fulfilled the BI-RADS 3 and 4 were enrolled in the study. Breast ultrasound with and without Doppler performed in these patients with GE Voluson S6 (Linear Array 4 - $12 \mathrm{MHz}$, Curved Array 2 - $8 \mathrm{MHz}$ ). Informed written consent for the ultrasound-guided biopsy procedures was obtained from each patient.

Patients who were unwilling to provide informed consent, Patients with breast implants and Patients with previous breast conserving surgery on the breast of interest were excluded from the study. 


\section{Ultrasound Examination}

The breast ultrasound examinations were performed using GE Voluson S6 (Linear Array 4 - 12 MHz, Curved Array 2 - 8 MHz) With patient in supine position and ipsilateral arm above the head, the affected breast was systematically examined with overlapping scans in transverse and radial planes from the nipple to the periphery to ensure complete coverage of all breast tissue. A minimum of two orthogonal B-mode images and one power or colour Doppler image was acquired. Maximum lesion diameter was measured on B mode images. The size, shape, margins, echotexture, posterior acoustic shadowing/enhancement, calcification and internal vascularity were evaluated in each of the cases as follows.6,7

- Border of the Lesion- A well-defined smooth, lobulated (Benign), ill-defined, irregular (Malignant).

- Orientation- Unique to US-imaging and defined as parallel (benign) or not parallel (Suspicious finding) to the skin.

- Echo Pattern- Anechoic, hypoechoic, complex cystic and solid, isoechoic, hyperechoic and heterogeneous. Echogenicity can contribute to the assessment of a lesion, together with other feature categories. Alone, it has little specificity.

- Posterior Features- Enhancement, shadowing. Posterior features represent the attenuation characteristics of a mass with respect to its acoustic transmission, also of additional value. Alone, it has little specificity.

- Calcifications- On US poorly characterised compared with mammography, but can be recognised as echogenic foci, particularly when in a mass.

- Associated Features- Architectural distortion, duct changes, skin changes, oedema and increased vascularity favours malignant lesion.

\section{Reference Standard}

The final diagnosis for each lesion was derived from histopathologic results after core biopsy. Positive reference standard was defined as malignant histopathologic (any invasive malignancy or ductal carcinoma in situ) result. The sensitivity, specificity and positive predictive value and negative predictive value of Ultrasound in predicting benign and malignancy were calculated.

All the data were analysed using Test statistics (Screening method)- Specificity. Sensitivity, Positive predictive value, negative predictive value and probability calculation.

\section{RESULTS}

A hundred and forty three patients with a single breast mass, each were included in the study. The mean age of our study population was 45.3 years \pm 13.6 years; (range 22 - 65 years). Seven patients were asymptomatic at first presentation, while the rest $(136 / 143,95.1 \%)$ presented with symptoms such as a palpable mass $(109 / 143,76.2 \%)$, breast pain $(46 / 143$, $32.1 \%)$, bloody nipple discharge $(9 / 143,6.3 \%)$, skin changes $(15 / 143 ; 12.6 \%)$ and nipple retraction $(7 / 143 ; 5.8 \%)$. The mean duration of symptoms was $6.5 \pm 5$ months (range, 1 - 36 months). The mean size of the breast mass in these patients was $2.3 \pm 1.4 \mathrm{~cm}$ (range, $0.5-9.4 \mathrm{~cm}$ ).

All the 143 masses were subjected to ultrasound-guided core biopsy and histopathological diagnosis was considered as the gold standard. Sixty four masses were benign, while seventy nine masses were malignant on histopathology. On comparison of the profile of patients with benign and malignant masses, the mean age of patients with benign masses was relatively less as compared to the ones with malignant disease $(35.2 \pm 11.7$ years vs $48.8 \pm 11.9$ years respectively, $\mathrm{p}<0.001)$. Significant differences were seen in symptoms and their duration in the two groups of patients. Palpable breast lump ( $96.7 \%$ vs $75.4 \%$ ), skin changes $(21 \%$ vs $3.5 \%)$ and nipple retraction $(11.3 \%$ vs $0 \%)$ were more common in patients with malignant masses, while pain was a common feature with patients with benign masses $(31.6 \%$ vs $14.5 \%, \mathrm{p}=0.02$ ). Mean duration of the symptoms was more in benign masses ( $7.2 \pm 6.4$ months) in comparison to malignant masses $(4 \pm 2.3$ months, $\mathrm{p}=0.001)$. The malignant masses had a larger mean size compared with those of the benign masses $(2.9 \pm 1.3 \mathrm{~cm}$ vs $2.5 \pm 1.5 \mathrm{~cm}$, respectively, $\mathrm{p}=0.03)$.

All the 143 breast masses were evaluated and categorised according to the BI-RADS classification on B mode US. A total of 51 masses were categorised into BI-RADS 3 and 92 masses into BI-RADS 4 . Among the 51 BI-RADS category 3 masses, only $4(7.8 \%)$ were malignant. Of the 92 masses of category 4 , 75 (81.5\%) were malignant on histopathology. Thus, the B mode US had a sensitivity of $94.9 \%$ and specificity of $73.4 \%$, positive predictive value of $81.5 \%$ and negative predictive value of $92.2 \%$. (P value $<0.001$ ) in detecting malignancy.

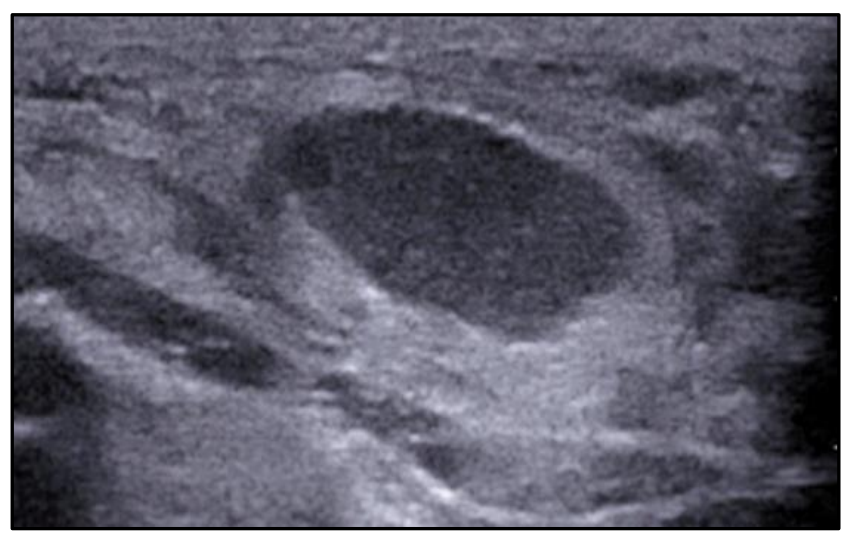

Figure 1. BI-RADS 3 Mass. Breast Ultrasound Images showing a Well Circumscribed Hypoechoic Mass with Smooth Margins with Posterior Acoustic Enhancement

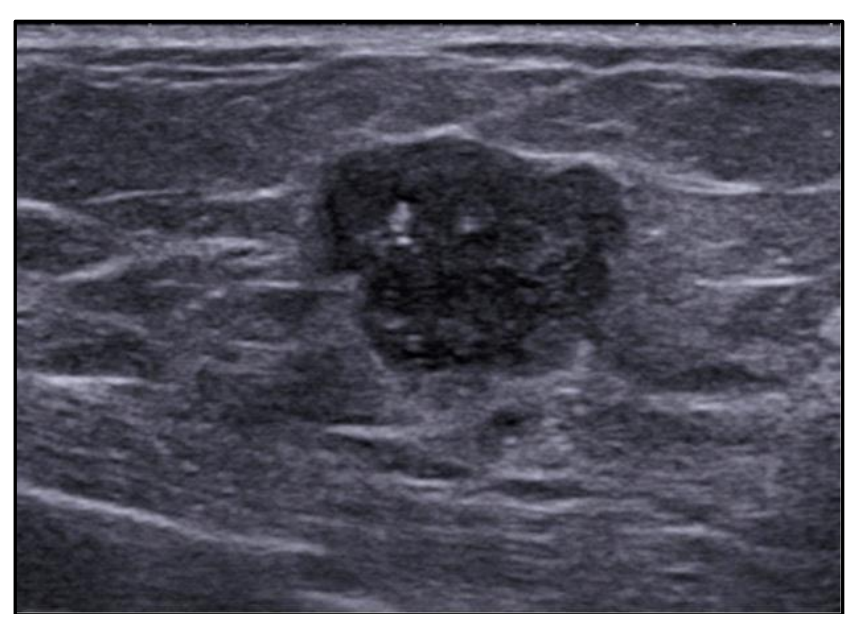

Figure 2. BIRADS 4 Mass- Breast Ultrasound Images showing a Hypoechoic Mass with Multiple Lobulations and Small Calcific Foci within It 


\begin{tabular}{|c|c|c|c|}
\hline \multirow{2}{*}{ USG Findings } & \multicolumn{2}{|c|}{ Biopsy Findings } & \multirow{2}{*}{ No. of Patients } \\
\cline { 2 - 4 } & Malignant & Benign & \\
\hline BIRADS 4 & 75 & 17 & 92 \\
\hline BIRADS 3 & 4 & 47 & 51 \\
\hline Total & $\mathbf{7 9}$ & $\mathbf{6 4}$ & $\mathbf{1 4 3}$ \\
\hline \multicolumn{3}{|c}{ Table 1. Correlation of USG and Biopsy Findings } \\
\hline
\end{tabular}

\section{DISCUSSION}

B mode US is the most widely used and invariably the first imaging modality for breast masses with a reported sensitivity of $69.6 \%$ to $100 \%$ and specificity of $14.3 \%$ to $94.8 \%(8,9,10,11,12,13,14)$ for the characterisation of these masses.

Our study population of 143 patients comprised of a mean age of $42.3 \pm 13.6$ years, predominantly females and the vast majority were symptomatic, the commonest of which was the presence of a breast lump seen in $76.5 \%$ followed by breast pain $(32.6 \%)$, skin changes (10.6\%), bloody nipple discharge (6.4\%) and nipple retraction (5.8\%). The mean size of the malignant breast masses was larger than that of the benign (2.4 \pm 1.3 vs $1.5 \pm 1.1 \mathrm{~cm}$, respectively). These presenting features reflect presence of chronic, longstanding disease of advanced nature at the outset.

In our study using the BI-RADS classification and considering BI-RADS 3 as benign and BI-RADS 4 as malignant, ultrasound had a high sensitivity of $94.9 \%$ and moderate specificity of $73.4 \%$ in detecting malignancy. The sensitivity of B mode ultrasound in detecting malignancy in our study (94.9\%) was consistent with most of the studies (Berg et al97.2\%, Lee et al-94.3\%, Evans et al-95\%).(10,11,13) On the other hand, the specificity of breast ultrasound was highly variable among different studies from $14.3 \%$ to $94.8 \%$. Our study showed a moderate specificity of $73.4 \%$, quite similar to the ones reported by Berg et al, Evans et al and Chang et al.(10,11,12) This variability could be attributed to the differences in the study population, prevalence of disease (pretest probability) and in the thresholds adopted for diagnosing malignancy on breast ultrasound. Due to lack of facility of the screening programs, all our patients were symptomatic and $76.2 \%$ had palpable breast lumps. A study conducted on similar population has yielded comparable results.(14)

We analysed the malignancy rates in our patient population according to the BI-RADS classification on B Mode US. The malignancy rate was $7.8 \%$ for BI-RADS 3 lesions and $81.5 \%$ for BI-RADS 4 masses.

\section{Limitations of the Study}

Diagnosis of breast masses in our study were based on ultrasound-guided core-needle biopsy. Although, 14-gauge ultrasound-guided core-needle biopsy has been proven to be a safe and accurate diagnostic method, false-negative rates of ultrasound-guided core-needle biopsy have been reported to range from $2.4 \%$ to $3.7 \%$.(15,16) There were limited spectrum of pathologies in our study. Studies involving larger number of patients with different histopathological diagnosis could help better assessment of the role of ultrasound.

\section{CONCLUSION}

Breast ultrasonography is an appropriate tool in the detection of cancer and should be included in the workup of symptomatic breast disease. Breast ultrasound has a very high sensitivity in detecting malignancies and guiding further management of the suspicious lesions. But the occurrence of false positive diagnosis in the clinical settings like that of inflammatory breast disease should also be considered and the management be decided after correlation with the clinical data and the biopsy results.

\section{REFERENCES}

[1] Bassett LW. Imaging of breast masses. Radiologic Clinics of North America 2000;38(4):669-91.

[2] Kaiser JS, Helvie MA, Blacklaw RL, et al. Palpable breast thickening: role of mammography and US in cancer detection. Radiology 2002;223(3):839-44.

[3] Moy L, Slanetz PJ, Moore R, et al. Specificity of mammography and US in the evaluation of a palpable abnormality: retrospective review. Radiology 2002;225(1):176-81.

[4] Hild F, Duda VF, Albert U, et al. Ductal orientated sonography improves the diagnosis of pathological nipple discharge of the female breast compared with galactography. European Journal of Cancer Prevention 1998;7 Suppl 1:S57-62.

[5] Parker SH, Burbank F, Jackman RJ, et al. Percutaneous large-core breast biopsy: a multi-institutional study. Radiology 1994;193(2):359-64.

[6] Mendelson EB, Berg WA, Merritt CR. Toward a standardized breast ultrasound lexicon, BI-RADS: ultrasound. Semin Roentgenol 2001;36(3):217-25.

[7] Stavros AT, Thickman D, Rapp CL, et al. Solid breast nodules: use of sonography to distinguish between benign and malignant lesions. Radiology 1995;196(1):123-34.

[8] Lazarus E, Mainiero MB, Schepps B, et al. BI-RADS lexicon for US and mammography: interobserver variability and positive predictive value. Radiology 2006;239(2):385-91.

[9] Youk JH, Gweon HM, Son EJ, et al. Diagnostic value of commercially available shear-wave elastography for breast cancers: integration into BI-RADS classification with subcategories of category 4. Eur Radiol 2013;23(10):2695-704.

[10] Berg WA, Cosgrove DO, Dore CJ, et al. Shear-wave elastography improves the specificity of breast US: the BE1 multinational study of 939 masses. Radiology 2012;262(2):435-49.

[11] Evans A, Whelehan P, Thomson K, et al. Differentiating benign from malignant solid breast masses: value of shear wave elastography according to lesion stiffness combined with greyscale ultrasound according to BIRADS classification. Br J Cancer 2012;107(2):224-9.

[12] Chang JM, Won JK, Lee KB, et al. Comparison of shearwave and strain ultrasound elastography in the differentiation of benign and malignant breast lesions. AJR Am J Roentgenol 2013;201(2):W347-56.

[13] Lee EJ, Jung HK, Ko KH, et al. Diagnostic performances of shear wave elastography: which parameter to use in differential diagnosis of solid breast masses? Eur Radiol 2013;23(7):1803-11.

[14] Zhou JQ, Zhan WW, Chang C, et al. Breast lesions: evaluation with shear wave elastography, with special emphasis on the "stiff rim" sign. Radiology 2014;272(1):63-72. 


\section{Jemds.com}

[15] Youk JH, Kim EK, Kim MJ, et al. Analysis of falsenegative results after US-guided 14-gauge core needle breast biopsy. Eur Radiol 2010;20(4):782-9.
Original Research Article

[16] Crystal P, Koretz M, Shcharynsky S, et al. Accuracy of sonographically guided 14-gauge core-needle biopsy: results of 715 consecutive breast biopsies with at least two-year follow-up of benign lesions. J Clin Ultrasound 2005;33(2):47-52. 